\title{
The Ingestion of a Fructose-Containing Beverage Combined with Fat Cream Exacerbates Postprandial Lipidemia in Young Healthy Women
}

\author{
Hiromi Saito ${ }^{1,2}$, Maiko Kato ${ }^{1}$, Akihiro Yoshida ${ }^{3}$ and Michitaka Naito ${ }^{1}$ \\ ${ }^{1}$ Division of Nutrition \& Health, School \& Graduate School of Life Studies, Sugiyama Jogakuen University, Nagoya, Japan \\ ${ }^{2}$ Department of Food Science and Nutrition, Nara Women's University, Nara, Japan \\ ${ }^{3}$ Department of Clinical Laboratory, Nakatsugawa Municipal General Hospital, Nakatsugawa, Japan
}

\begin{abstract}
Aim: To investigate the acute effects of the ingestion of a fructose-containing beverage combined with fat on postprandial lipoprotein metabolism.

Methods: Twelve young healthy Japanese women with apolipoprotein E phenotype 3/3 were enrolled in this study. At each of four sessions, the subjects ingested one of four sugar beverages containing fructose and/or glucose (total: $0.5 \mathrm{~g} / \mathrm{kg}$ body weight) combined with OFTT cream $(1 \mathrm{~g} / \mathrm{kg}, 0.35 \mathrm{~g} / \mathrm{kg}$ as fat) in a randomized crossover design. The four sugar beverages were as follows: $100 \%(\mathrm{w} / \mathrm{w})$ fructose (F100), 90\% fructose $+10 \%$ glucose (F90G10), 55\% fructose $+45 \%$ glucose (F55G45) and $100 \%$ glucose (G100). Venous blood samples were obtained at baseline and 0.5 , one, two, four and six hours after ingestion.

Results: The serum concentrations of TG in the F100, F90G10 and F55G45 trials were significantly higher than each fasting value at two and four hours, and returned to baseline at six hours, except in the F100 trial. The concentrations at four hours and the incremental areas under the curve for the hepatic triglyceride-rich lipoprotein-triglyceride (VLDL-TG ${ }^{\mathrm{TM}}$ ) levels in the F100 and F90G10 trials were significantly higher and larger, respectively, than those observed in the G100 trial. Meanwhile, the concentrations of RLP-TG and apolipoprotein B-48 peaked at two hours in the G100 trial, versus four hours in the other trials, and did not return to baseline at six hours, except in the G100 trial. At four hours, the $\triangle \mathrm{apoB} 48$ tended to be higher in the F100 trial than in the G100 trial.

Conclusions: The ingestion of a high-fructose-containing beverage with fat cream delays the clearance of chylomicron and its remnant derived from the intestine and enhances the secretion of triglyceride-rich lipoprotein particles from the liver, thereby inducing postprandial lipidemia, even in young healthy women.
\end{abstract}

J Atheroscler Thromb, 2015; 22:85-94.

Key words: Fructose, Fat, Postprandial lipidemia, Triglyceride-rich lipoprotein, Apolipoprotein B-48

\section{Introduction}

The consumption of high-fructose corn syrup (HFCS) as an alternative to sucrose has been increasing in Japan since 1980. Soft drinks account for

Address for correspondence: Michitaka Naito, Division of Nutrition \& Health, School \& Graduate School of Life Studies, Sugiyama Jogakuen University, 17-3, Hoshigaokamotomachi, Chikusa-ku, Nagoya 464-8662, Japan

E-mail: naito@sugiyama-u.ac.jp

Received: November 22, 2013

Accepted for publication: July 23, 2014 approximately $50 \%$ of the total consumption of HFCS $^{1)}$. HFCS is classified into three different types: high-fructose liquid sugar (fructose $\geq 90 \%$ ), fructoseglucose liquid sugar $(50 \% \leq$ fructose $<90 \%)$ and glucose-fructose liquid sugar (fructose $<50 \%$ ) ) $^{2}$. Of these, the most commonly used is fructose-glucose liquid sugar $^{3)}$.

The overconsumption of fructose can have adverse effects on human health. Diets containing $>15 \%$ of energy derived from fructose, as compared with glucose, are consistently associated with increases in both the fasting and postprandial triglyceride (TG) 
concentrations $s^{4,5)}$, and postprandial hyperlipidemia is widely accepted to be a major risk factor for coronary heart disease, as first proposed by Zilversmit ${ }^{6}$. Furthermore, the habitual consumption of fructose has also been reported to increase the deposition of visceral adipose tissue ${ }^{7)}$. In our previous study, the serum concentrations of TG, remnant-like particle (RLP)TG and apolipoprotein B-48 (apoB48) were significantly increased following the ingestion of a moderate amount of fructose $(0.5 \mathrm{~g} / \mathrm{kg}$ body weight $)$ combined with fat compared to that observed after the ingestion of glucose in healthy young women, even in a single ingestion, demonstrating that fructose markedly exacerbates postprandial exogenous lipidemia ${ }^{8)}$.

In our previous study, the subjects ingested a beverage containing fructose (without glucose) combined with fat cream. In reality, however, fructose is usually consumed as HFCS in soft drinks and many other foods ${ }^{9)}$. In the present study, we enrolled young healthy women, thought to be among the highest consumers of fructose, and investigated TRL changes following the ingestion of fructose and/or glucose combined with fat cream.

\section{Aim}

The aim of this study was to elucidate the acute effects of the ingestion of a fructose-containing beverage combined with fat cream on postprandial exogenous and endogenous lipoprotein metabolism in young healthy Japanese women.

\section{Methods}

\section{Subjects}

Twelve young healthy Japanese women with a normal ovarian cycle and apolipoprotein E (apoE) phenotype $3 / 3$ were enrolled as participants. All subjects were non-smokers, had no apparent acute or chronic illnesses and were not taking any medications or dietary supplements. This study was approved by the Ethics Committee of the Sugiyama Jogakuen University School of Life Studies, and each subject provided their written informed consent for study participation.

\section{Anthropometric and Body Composition Measure- ments}

Body weight and height were measured according to standard methods. Waist circumference was assessed as the abdominal girth at the level of the umbilicus, and hip circumference was measured at the level of the greater trochanters. The waist-to-hip (W/
H) ratio was also calculated. Body composition, including the visceral fat area (VFA), was analyzed according to the eight-polar bioelectrical impedance method using the InBody720 device (Biospace, Tokyo, Japan).

\section{Sugar and Fat Load Tests}

Each subject underwent four test trials. In each test trial, the subjects ingested one of four beverages containing fructose $(\mathrm{F})$ and/or glucose $(\mathrm{G})$ (total: 0.5 $\mathrm{g} / \mathrm{kg}$ body weight) combined with oral fat tolerance test (OFTT) cream (Jomo, Takasaki, Japan; $1 \mathrm{~g} / \mathrm{kg}$, $0.35 \mathrm{~g} / \mathrm{kg}$ as fat) in a randomized crossover design. The OFTT cream was used as previously described ${ }^{8,10-13)}$. The four beverages were prepared as follows: F100: $100 \%(w / w)$ fructose (Nisshin Seito, Tokyo, Japan), F90G10: 90\% fructose + 10\% glucose (Fuso Pharmaceutical, Osaka, Japan), F55G45: 55\% fructose $+45 \%$ glucose and G100: 100\% glucose.

\section{Experimental Design}

The subjects abstained from consuming caffeine or alcohol on the day before the experiment and ingested one of the four beverages after a 12-hour overnight fast. Venous blood samples were obtained before (0 hours) and at 0.5 , one, two, four and six hours after ingestion. During the tests, the subjects avoided exercise and eating, although they were granted free access to water after one hour. All blood samples were obtained with the participant in the supine position. An interval of four weeks was observed between the test days in order to minimize the confounding effects of the subject's menstrual status on lipid metabolism.

\section{Biochemical Analysis}

The serum samples were immediately refrigerated at $4{ }^{\circ} \mathrm{C}$ or frozen at $-80^{\circ} \mathrm{C}$ until the analysis. The level of fructose was measured enzymatically (BioAssay Systems, CA, USA). The level of glucose was measured according to the mutarotase GOD method (Wako, Osaka, Japan). The level of insulin was measured using a chemiluminescent enzyme immunoassay (Fujirebio, Tokyo, Japan). Insulin resistance was evaluated according to the homeostasis model assessment for insulin resistance (HOMA-IR) ${ }^{14)}$. The hemoglobin A1c (HbA1c) level was measured using the latex agglutination method (Fujirebio) and expressed as the National Glycohemoglobin Standardization Program (NGSP) value. The levels of free fatty acids (FFA) (Eiken Chemical, Tokyo, Japan) and lactate (Kyowa Medex, Tokyo, Japan) were measured enzymatically. The level of $\beta$-hydroxybutyric acid $(\beta-\mathrm{HB})$ was mea- 
Table 1. Anthropometric and clinical characteristics

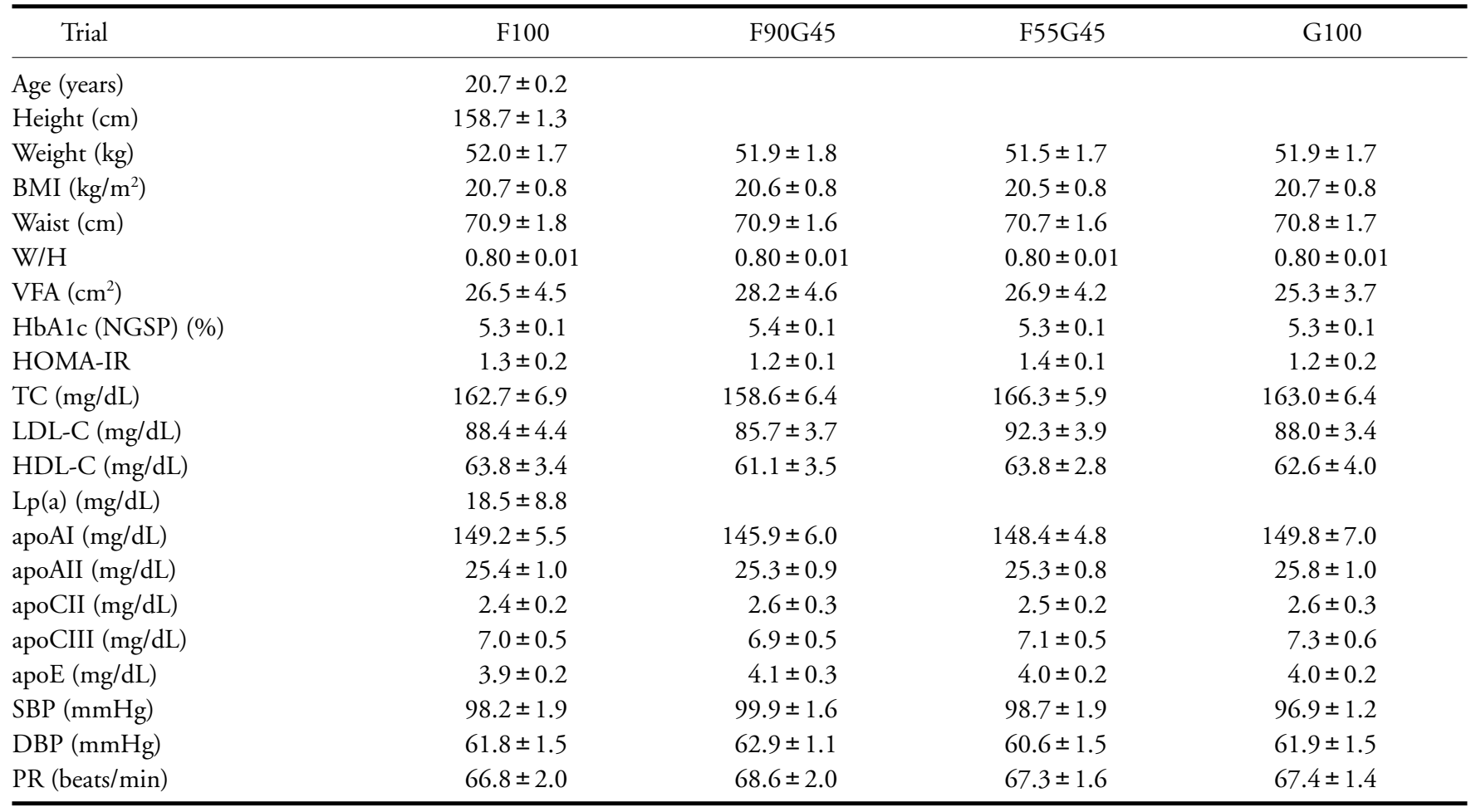

All values are presented as the mean \pm SEM.

SBP: systolic blood pressure, DBP: diastolic blood pressure, PR: pulse rate

sured using an enzymatic cycling method (Kainos, Tokyo, Japan). The level of total cholesterol (TC) was measured enzymatically (Sysmex, Hyogo, Japan). The level of high-density lipoprotein-cholesterol (HDL-C) was measured using a direct method (Fujirebio), while the level of low-density lipoprotein-cholesterol (LDLC) was calculated according to the Friedewald formula. The level of TG was measured enzymatically (Sekisui Medical, Tokyo, Japan).

The TG concentration in the TG-rich lipoproteins was estimated using a newly developed homogenous assay (VLDL-TG ${ }^{\mathrm{TM}}$, Shino-test, Tokyo, Japan) ${ }^{15)}$ and presented as the hepatic triglyceride-rich lipoproteintriglyceride (hTRL-TG) value. The level of RLP-TG was measured using an immunosorbent assay (Otsuka Pharmaceutical, Tokyo, Japan), and the level of remnant lipoprotein-cholesterol (RemL-C) was determined using a homogenous assay (MetaboRead ${ }^{\mathrm{TM}}$, Kyowa Medex, Japan). The level of lipoprotein(a) $(\mathrm{Lp}(\mathrm{a}))$ was measured according to the latex agglutination method (Sekisui Medical), and the levels of apolipoproteins AI, AII, B(100), CII, CIII and E were measured using the immunoturbidimetric method (Sekisui Medical). The level of apoB48 was measured according to a chemiluminescent enzyme immunoas- say (Fujirebio). The apoE phenotype was determined using the isometric electrophoresis method (Phenotyping ApoE IEF System ${ }^{\text {TM }}$, Joko, Tokyo, Japan).

\section{Quantification of Postprandial Metabolism}

Postprandial changes in the concentrations of TG, hTRL-TG, RLP-TG, RemL-C, apoB and apoB48 were calculated as the difference from the baseline mean value (as 0 at 0 hours) and shown as $\triangle \mathrm{TG}, \triangle \mathrm{hTRL}$ TG, $\Delta$ RLP-TG, $\Delta$ RemL-C, $\Delta$ apoB and $\Delta$ apoB 48 , respectively. The postprandial metabolism was quantified by calculating the incremental area under the curve ( $\triangle \mathrm{AUC})$, which was defined as the difference between the area under the curve and the area below the baseline ( 0 hours) concentration from zero to six hours, as previously described ${ }^{8)}$.

\section{Statistics}

All data are expressed as the mean \pm SEM. The statistical analyses were performed using the SPSS ver. 19 software package (IBM, Tokyo, Japan). Differences in the time course compared with the fasting values were analyzed using the Friedman test, followed by the Wilcoxon signed-rank test with the Bonferroni correction. The differences in the values at each time 
Table 2. The values of fasting and postprandial fructose, glucose, insulin, FFA, $\beta-\mathrm{HB}$ and lactate

\begin{tabular}{|c|c|c|c|c|c|c|c|c|c|}
\hline Time (h) & 0 & 0.5 & & 1 & & 2 & & 4 & 6 \\
\hline \multicolumn{10}{|c|}{ Fructose $(\mathrm{mg} / \mathrm{dL})$} \\
\hline F100 & $0.72 \pm 0.06$ & $4.25 \pm 0.74^{*}$ & & $5.48 \pm 0.54^{*}$ & & $3.99 \pm 0.50^{*}$ & & $0.79 \pm 0.06$ & $0.83 \pm 0.08$ \\
\hline F90G10 & $0.63 \pm 0.05$ & $4.64 \pm 0.64^{*}$ & & $4.74 \pm 0.54^{*}$ & $\#$ & $2.75 \pm 0.25^{*}$ & $\#$ & $0.98 \pm 0.12^{*}$ & $0.85 \pm 0.06$ \\
\hline F55G45 & $0.92 \pm 0.07$ & $J^{\#} \quad 3.02 \pm 0.52^{*}$ & $7_{+} \#^{\prime}$ & $3.02 \pm 0.29^{*}$ & $\# \perp$ & $2.08 \pm 0.23^{*}$ & $\#\rfloor{ }^{\#}$ & $0.85 \pm 0.09$ & $0.69 \pm 0.07$ \\
\hline G100 & $0.62 \pm 0.08$ & $0.78 \pm 0.09$ & ]\# & $0.54 \pm 0.08$ & & $0.86 \pm 0.06$ & . & $0.65 \pm 0.07$ & $0.74 \pm 0.07$ \\
\hline \multicolumn{10}{|c|}{ Glucose (mg/dL) } \\
\hline F100 & $84.0 \pm 1.5$ & $88.9 \pm 1.9$ & & $86.4 \pm 1.7$ & & $87.8 \pm 1.7$ & & $84.1 \pm 1.4$ & $80.8 \pm 1.8$ \\
\hline F90G10 & $85.0 \pm 1.1$ & $91.5 \pm 1.8^{*}$ & & $87.3 \pm 1.6$ & & $87.3 \pm 1.2$ & & $83.0 \pm 1.1$ & $80.1 \pm 1.1^{*}$ \\
\hline F55G45 & $86.3 \pm 1.2$ & $101.7 \pm 4.3$ & & $92.6 \pm 4.0$ & & $89.6 \pm 2.0$ & & $82.9 \pm 1.7$ & $80.4 \pm 1.3^{*}$ \\
\hline G100 & $85.6 \pm 1.5$ & $106.7 \pm 4.5^{*}$ & & $99.1 \pm 4.9$ & & $93.0 \pm 3.9$ & & $82.9 \pm 1.7$ & $80.1 \pm 1.4^{*}$ \\
\hline \multicolumn{10}{|c|}{ Insulin $(\mu \mathrm{U} / \mathrm{mL})$} \\
\hline F100 & $6.23 \pm 0.75$ & $13.93 \pm 1.44^{*}$ & \multirow{4}{*}{ \# } & $12.57 \pm 1.98^{*}$ & \multirow{4}{*}{$\#$} & $9.69 \pm 0.96^{*}$ & & $4.12 \pm 0.50^{*}$ & $3.29 \pm 0.54^{*}$ \\
\hline F90G10 & $5.71 \pm 0.54$ & $19.31 \pm 2.42^{*}$ & & $12.55 \pm 1.52^{*}$ & & $10.39 \pm 1.16^{*}$ & & $3.54 \pm 0.34^{*}$ & $3.33 \pm 0.56^{*}$ \\
\hline F55G45 & $6.48 \pm 0.70$ & $30.84 \pm 6.53^{*}$ & & $18.80 \pm 3.19^{*}$ & & $9.82 \pm 1.04^{*}$ & & $5.03 \pm 1.06$ & $3.89 \pm 0.73^{*}$ \\
\hline G100 & $5.79 \pm 0.89$ & $36.98 \pm 6.32^{*}$ & & $31.31 \pm 6.10^{*}$ & & $11.87 \pm 2.00^{*}$ & & $3.64 \pm 0.41^{*}$ & $3.28 \pm 0.42^{*}$ \\
\hline \multicolumn{10}{|c|}{ FFA $(\mu \mathrm{mol} / \mathrm{L})$} \\
\hline F100 & $426.3 \pm 38.4$ & & & $266.6 \pm 44.3$ & & $256.2 \pm 34.9^{*}$ & & $608.8 \pm 46.1^{*}$ & $776.3 \pm 38.7^{*}$ \\
\hline F90G10 & $495.7 \pm 49.4$ & & & $255.4 \pm 32.2^{*}$ & & $256.6 \pm 35.4^{*}$ & & $605.3 \pm 34.8$ & $730.3 \pm 32.0^{*}$ \\
\hline F55G45 & $511.8 \pm 50.9$ & & & $255.6 \pm 36.2^{*}$ & & $280.2 \pm 29.5^{*}$ & & $700.2 \pm 39.8^{*}$ & $852.3 \pm 48.1^{*}$ \\
\hline G100 & $460.3 \pm 52.4$ & & & $173.8 \pm 19.5^{*}$ & & $178.7 \pm 22.5^{*}$ & & $673.3 \pm 45.3^{*}$ & $825.4 \pm 47.2^{*}$ \\
\hline \multicolumn{10}{|c|}{$\beta-\mathrm{HB}(\mu \mathrm{mol} / \mathrm{L})$} \\
\hline F100 & $63.4 \pm 9.6$ & & & $49.9 \pm 9.5$ & & $33.0 \pm 3.7$ & & $175.8 \pm 35.7^{*}$ & $294.5 \pm 41.1^{*}$ \\
\hline F90G10 & $73.4 \pm 15.2$ & & & $47.4 \pm 9.3$ & & $31.7 \pm 3.3^{*}$ & & $156.0 \pm 22.4^{*}$ & $288.4 \pm 34.8^{*}$ \\
\hline F55G45 & $87.3 \pm 26.0$ & & & $54.7 \pm 10.9$ & & $46.3 \pm 8.0$ & & $232.3 \pm 31.9^{*}$ & $355.8 \pm 42.2^{*}$ \\
\hline G100 & $69.2 \pm 17.8$ & & & $54.7 \pm 11.2$ & & $35.4 \pm 4.4$ & & $219.4 \pm 36.4^{*}$ & $377.6 \pm 80.0^{*}$ \\
\hline \multicolumn{10}{|c|}{ Lactate $(\mathrm{mg} / \mathrm{dL})$} \\
\hline F100 & $9.3 \pm 0.6$ & & & $15.9 \pm 1.8^{*}$ & & $13.8 \pm 1.0^{*}$ & \multirow{4}{*}{ \# } & $8.4 \pm 0.5$ & $8.3 \pm 0.6$ \\
\hline F90G10 & $9.9 \pm 0.6$ & & & $15.9 \pm 1.3^{*}$ & & $12.2 \pm 0.6$ & & $8.7 \pm 0.6$ & $8.4 \pm 0.5$ \\
\hline F55G45 & $10.2 \pm 0.6$ & & & $13.9 \pm 1.0$ & & $11.9 \pm 0.8$ & & $8.9 \pm 0.5$ & $8.8 \pm 0.4$ \\
\hline G100 & $10.5 \pm 1.0$ & & & $11.3 \pm 0.7$ & & $9.9 \pm 0.5$ & & $9.0 \pm 0.5$ & $8.8 \pm 0.5$ \\
\hline
\end{tabular}

All values are presented as the mean \pm SEM. ${ }^{*} p<0.05$ compared to the fasting value in each trial. ${ }^{\#} p<0.05$ compared between the trials.

point in the four trials were assessed using the Kruskal-Wallis test, followed by the Mann-Whitney $U$-test with the Bonferroni correction.

\section{Results}

The physical characteristics and fasting blood chemistry data of the subjects are shown in Table $\mathbf{1}$. There were no significant differences in any of the physical characteristics among the four trials.

\section{Fructose, Glucose, Insulin, FFA, $\beta$-HB and Lactate}

The serum concentrations of fasting and postprandial fructose, glucose, insulin, FFA, $\beta-\mathrm{HB}$ and lactate in the four trials are presented in Table 2.

The serum fructose concentrations in the F100, F90G10 and F55G45 trials were significantly higher than those observed in the G100 trial at 0.5 , one and two hours $(p<0.05$ each). In contrast, there were no significant differences in the serum fructose concentrations among the four trials at four or six hours.

The serum concentrations of glucose were significantly increased at 0.5 hours in the G100 and F90G10 trials compared with the respective fasting values $(p<0.05$ each). The glucose concentrations in the G100 trial tended to be higher than those observed in the F100 $(p=0.06)$ and F90G10 $(p=0.15)$ trials at 0.5 hours. There were no significant differ- 
Table 3. The values of fasting and postprandial TG, hTRL-TG, RLP-TG, RemL-C, apoB and apoB48

\begin{tabular}{|c|c|c|c|c|c|}
\hline Time (h) & 0 & 1 & 2 & 4 & 6 \\
\hline \multicolumn{6}{|l|}{ TG (mg/dL) } \\
\hline F100 & $52.3 \pm 3.8$ & $55.4 \pm 3.8$ & $64.3 \pm 4.6^{*}$ & $94.8 \pm 10.3^{*}$ & $66.3 \pm 4.8^{*}$ \\
\hline F90G10 & $59.3 \pm 8.6$ & $60.6 \pm 7.9$ & $70.8 \pm 9.5^{*}$ & $101.2 \pm 17.5^{*}$ & $68.7 \pm 8.7$ \\
\hline F55G45 & $51.6 \pm 5.6$ & $54.6 \pm 4.5$ & $63.8 \pm 4.4^{*}$ & $76.2 \pm 10.2^{*}$ & $54.9 \pm 5.7$ \\
\hline G100 & $62.3 \pm 5.0$ & $67.7 \pm 4.5$ & $78.2 \pm 6.7$ & $79.3 \pm 8.6$ & $60.4 \pm 5.2$ \\
\hline \multicolumn{6}{|c|}{ hTRL-TG (mg/dL) } \\
\hline F100 & $39.6 \pm 4.5$ & $43.8 \pm 4.2$ & $48.9 \pm 4.2$ & $68.1 \pm 7.5^{*}$ & $50.2 \pm 4.8^{*}$ \\
\hline F90G10 & $46.8 \pm 7.7$ & $48.3 \pm 7.5$ & $53.1 \pm 8.2^{*}$ & $69.9 \pm 9.7^{*}$ & $52.0 \pm 7.4$ \\
\hline F55G45 & $40.7 \pm 6.0$ & $43.0 \pm 4.9$ & $46.4 \pm 4.6$ & $55.2 \pm 7.8^{*}$ & $41.5 \pm 5.4$ \\
\hline G100 & $52.1 \pm 5.8$ & $56.2 \pm 5.4$ & $55.6 \pm 5.1$ & $58.2 \pm 6.8$ & $44.8 \pm 4.6$ \\
\hline \multicolumn{6}{|c|}{ RLP-TG (mg/dL) } \\
\hline F100 & $8.6 \pm 0.9$ & $11.2 \pm 1.1$ & $17.5 \pm 2.2^{*}$ & $30.7 \pm 4.7^{*}$ & $15.5 \pm 1.7^{*}$ \\
\hline F90G10 & $9.4 \pm 1.3$ & $11.3 \pm 1.4$ & $18.2 \pm 2.6^{*}$ & $32.3 \pm 9.0^{*}$ & $14.7 \pm 2.3^{*}$ \\
\hline F55G45 & $8.5 \pm 0.7$ & $12.1 \pm 0.8^{*}$ & $18.5 \pm 1.7^{*}$ & $22.5 \pm 4.0^{*}$ & $12.0 \pm 0.9^{*}$ \\
\hline G100 & $9.6 \pm 0.9$ & $13.9 \pm 1.0^{*}$ & $23.5 \pm 3.4^{*}$ & $21.1 \pm 3.3^{*}$ & $13.7 \pm 1.6$ \\
\hline \multicolumn{6}{|c|}{$\operatorname{RemL}-\mathrm{C}(\mathrm{mg} / \mathrm{dL})$} \\
\hline F100 & $3.7 \pm 0.3$ & $3.9 \pm 0.4$ & $4.2 \pm 0.4$ & $6.1 \pm 0.7^{*}$ & $5.2 \pm 0.5^{*}$ \\
\hline F90G10 & $4.5 \pm 1.0$ & $4.4 \pm 0.9$ & $4.7 \pm 0.9$ & $6.4 \pm 1.2^{*}$ & $5.7 \pm 1.0^{*}$ \\
\hline F55G45 & $4.0 \pm 0.6$ & $4.0 \pm 0.5$ & $4.3 \pm 0.4$ & $5.4 \pm 0.7^{*}$ & $4.8 \pm 0.7^{*}$ \\
\hline G100 & $4.5 \pm 0.4$ & $4.7 \pm 0.3$ & $4.7 \pm 0.4$ & $5.3 \pm 0.5$ & $4.7 \pm 0.5$ \\
\hline \multicolumn{6}{|l|}{ apoB (mg/dL) } \\
\hline F100 & $63.2 \pm 2.8$ & $63.7 \pm 1.7$ & $61.3 \pm 2.6^{*}$ & $63.1 \pm 2.7$ & $63.5 \pm 2.5$ \\
\hline F90G10 & $62.6 \pm 2.5$ & $60.6 \pm 2.5^{*}$ & $60.2 \pm 2.4^{*}$ & $62.0 \pm 2.6$ & $62.5 \pm 2.6$ \\
\hline F55G45 & $65.0 \pm 2.8$ & $62.5 \pm 2.4^{*}$ & $62.3 \pm 2.6^{*}$ & $64.1 \pm 2.6$ & $64.4 \pm 2.8$ \\
\hline G100 & $64.5 \pm 2.2$ & $62.3 \pm 1.9^{*}$ & $61.0 \pm 1.8^{*}$ & $63.4 \pm 1.8$ & $63.9 \pm 2.0$ \\
\hline \multicolumn{6}{|c|}{ apoB48 $(\mu \mathrm{g} / \mathrm{mL})$} \\
\hline F100 & $2.3 \pm 0.3$ & $2.9 \pm 0.3$ & $4.0 \pm 0.5^{*}$ & $5.2 \pm 0.6^{*}$ & $3.4 \pm 0.2^{*}$ \\
\hline F90G10 & $2.4 \pm 0.6$ & $3.0 \pm 0.5$ & $4.0 \pm 0.6^{*}$ & $5.1 \pm 1.1^{*}$ & $3.6 \pm 0.6^{*}$ \\
\hline F55G45 & $2.1 \pm 0.1$ & $3.2 \pm 0.4^{*}$ & $3.8 \pm 0.3^{*}$ & $4.1 \pm 0.5^{*}$ & $3.3 \pm 0.4^{*}$ \\
\hline G100 & $2.7 \pm 0.4$ & $4.0 \pm 0.6^{*}$ & $4.4 \pm 0.5^{*}$ & $3.9 \pm 0.6^{*}$ & $3.2 \pm 0.4$ \\
\hline
\end{tabular}

All values are presented as the mean \pm SEM. ${ }^{*} p<0.05$ compared to the fasting value in each trial.

ences in the serum glucose concentrations among the four trials at one hour.

The serum concentrations of insulin were lower in the F100 and F90G10 trials than in the G100 trial at 0.5 hours (F100 vs. G100 $p<0.05$, F90G10 vs. $\mathrm{G} 100 p=0.09)$ and one hour (F100 and F90G10 vs. G100 $p<0.05)$.

The serum concentrations of FFA instantly decreased in the G100, F55G45 and F90G10 trials, reaching the lowest level at one hour in these trials versus two hours in the F100 trial, followed by an increase in all trials. At four and six hours, the serum FFA concentrations in the F100 and F90G10 trials were lower, although not significantly, than that observed in the G100 trial.

The serum $\beta-\mathrm{HB}$ concentrations at four and six hours in the four trials were significantly higher than each respective fasting value $(p<0.05$ each). At four and six hours, the serum $\beta-\mathrm{HB}$ concentrations in the F100 and F90G10 trials were lower, although not significantly, than that observed in the G100 trial.

The serum lactate concentrations in the F100 and F90G10 trials were increased at one hour $(p<$ 0.05 each) and two hours (F100 $p<0.05$, F90G10 $p=0.17)$ compared to the respective fasting values, then returned to baseline at four hours. At two hours, the serum lactate concentrations in the F100 $(p<$ $0.05)$ and F90G10 $(p=0.08)$ trials were higher than 
that observed in the G100 trial.

\section{TG, hTRL-TG, RLP-TG and RemL-C}

The concentrations of fasting and postprandial TG, hTRL-TG, RLP-TG and RemL-C in the four trials are presented in Table 3, and the time courses of $\triangle \mathrm{TG}, \triangle \mathrm{hTRL}-\mathrm{TG}, \Delta \mathrm{RLP}-\mathrm{TG}$ and $\triangle \mathrm{RemL}-\mathrm{C}$ are shown in Fig. 1A-D.

The $\Delta$ TG values in the F100, F90G10 and F55G45 trials were significantly higher than the respective fasting levels at two and four hours $(p<0.05)$ and then returned to the fasting levels at six hours, except in the F100 trial. The $\Delta \mathrm{TG}$ values in the G100 trial tended to be higher than the fasting levels at two hours $(p=0.11)$. In addition, the $\triangle \mathrm{AUC}-\mathrm{TG}$ values tended to be larger in the F100 trial than in the G100 $(p=0.09)$ and F55G45 ( $p=0.13)$ trials (Fig. 2A).

The $\triangle$ hTRL-TG values at two hours in the F100 $(p=0.06)$, F90G10 $(p<0.05)$ and F55G45 $(p=0.10)$ trials were higher than the respective fasting levels, peaking at four hours then returning to the fasting levels at six hours, except in the F100 trial. In contrast, the $\triangle \mathrm{hTRL}-\mathrm{TG}$ values in the G100 trial did not change significantly during six hours. Meanwhile, the $\triangle \mathrm{hTRL}$-TG values at four hours were significantly higher in the F100 and F90G10 trials than in the G100 trial $(p<0.05$ each). The $\triangle$ AUC-hTRL-TG values were significantly larger in the F100 trial than in the G100 and F55G45 trials $(p<0.05$ each) and significantly larger in the F90G10 trial than in the G100 trial $(p<0.05)$ (Fig. 2B).

The $\triangle \mathrm{RLP}-\mathrm{TG}$ values were significantly higher than the respective fasting levels at one hour in the F55G45 and G100 trials ( $p<0.05$ each) and two hours in the F100 and F90G10 trials ( $p<0.05$ each). In addition, the $\triangle \mathrm{RLP}-\mathrm{TG}$ values peaked at two hours in the G100 trial and at four hours in the other trials. In all trials, except for the G100 trial, the $\triangle \mathrm{RLP}-\mathrm{TG}$ values did not return to the respective fasting levels at six hours, while the $\triangle$ AUC-RLP-TG values exhibited no significant differences among the four trials (data not shown).

In all trials, except the G100 trial, the $\Delta$ RemL-C values were significantly increased at four and six hours ( $p<0.05$ each). In the G100 trial, the $\Delta$ RemL$\mathrm{C}$ values did not differ significantly from the fasting levels throughout the six-hour period. In contrast, the $\triangle$ RemL-C values in the F100 and F90G10 trials were higher than those observed in the G100 trial at four hours (F100 vs. G100 $p<0.05$, F90G10 vs. G100 $p=0.15$ ) and six hours (F100 vs. G100 $p<0.05$, F90G10 vs. G100 $p=0.06$ ), and the $\triangle$ AUC-RemL-C values in the $\mathrm{F} 100$ trial were larger than those noted in the G100 and F55G45 trials $(p<0.05$ each) (Fig. 2C).

\section{apoB and $\mathrm{apoB} 48$}

The concentrations of fasting and postprandial apoB and apoB48 in the four trials are presented in Table 3, and the time courses of the $\triangle \mathrm{apoB}$ and $\triangle$ apoB 48 values are shown in Fig. 1E \& F.

The $\triangle \mathrm{apoB}$ values in the F90G10, F55G45 and G100 trials were significantly decreased at both one and two hours, compared to only two hours in the F100 trial $(p<0.05$ each). In all four trials, the $\Delta$ apoB values returned to the fasting levels at four hours. Furthermore, the $\triangle \mathrm{AUC}$-apoB values did not differ between the four trials (data not shown).

The $\triangle$ apoB48 values in the F55G45 and G100 trials were significantly increased at one hour compared with the respective fasting values $(p<0.05$ each) and at two hours in the F100 and F90G10 trials $(p<$ 0.05 each). Meanwhile, the $\triangle \mathrm{apoB} 48$ values peaked at two hours in the G100 trial and at four hours in the other trials. The $\triangle$ apoB 48 values at four hours in the F100 trial tended to be higher than those observed in the G100 trial $(p=0.09)$. In all but the G100 trial, the $\triangle$ apoB48 values did not return to baseline at six hours. However, there were no significant differences in the $\triangle \mathrm{AUC}$-apoB48 values among the four trials (data not shown).

\section{Discussion}

We previously demonstrated that the ingestion of fructose combined with fat leads to a significantly higher rise (with a delayed peak) in the serum concentrations of TG, RLP-TG, RemL-C and apoB48 compared to the ingestion of glucose with fat, suggesting a delay in the removal of intestinal TG-rich lipoproteins (TRL), namely chylomicron (CM) and chylomicronremnant $(\mathrm{CM}-\mathrm{R})^{8}$. In that study, we examined the effects of pure fructose combined with fat on postprandial lipoprotein metabolism. However, in daily dietary habits, fructose is usually consumed with glucose in the form of HFCS or sucrose, such as that contained in colas and other soft drinks. Therefore, in the present study, we examined the effects of the mixed ingestion of fructose and glucose (as HFCS) combined with fat on postprandial lipoprotein and carbohydrate metabolism.

The major finding of the present study is that the ingestion of high-fructose- (but not glucose-) containing beverages combined with fat cream (the F100 and F90G10 trials) exacerbated both exogenous (intestinal) and endogenous (hepatic) lipidemia post- 

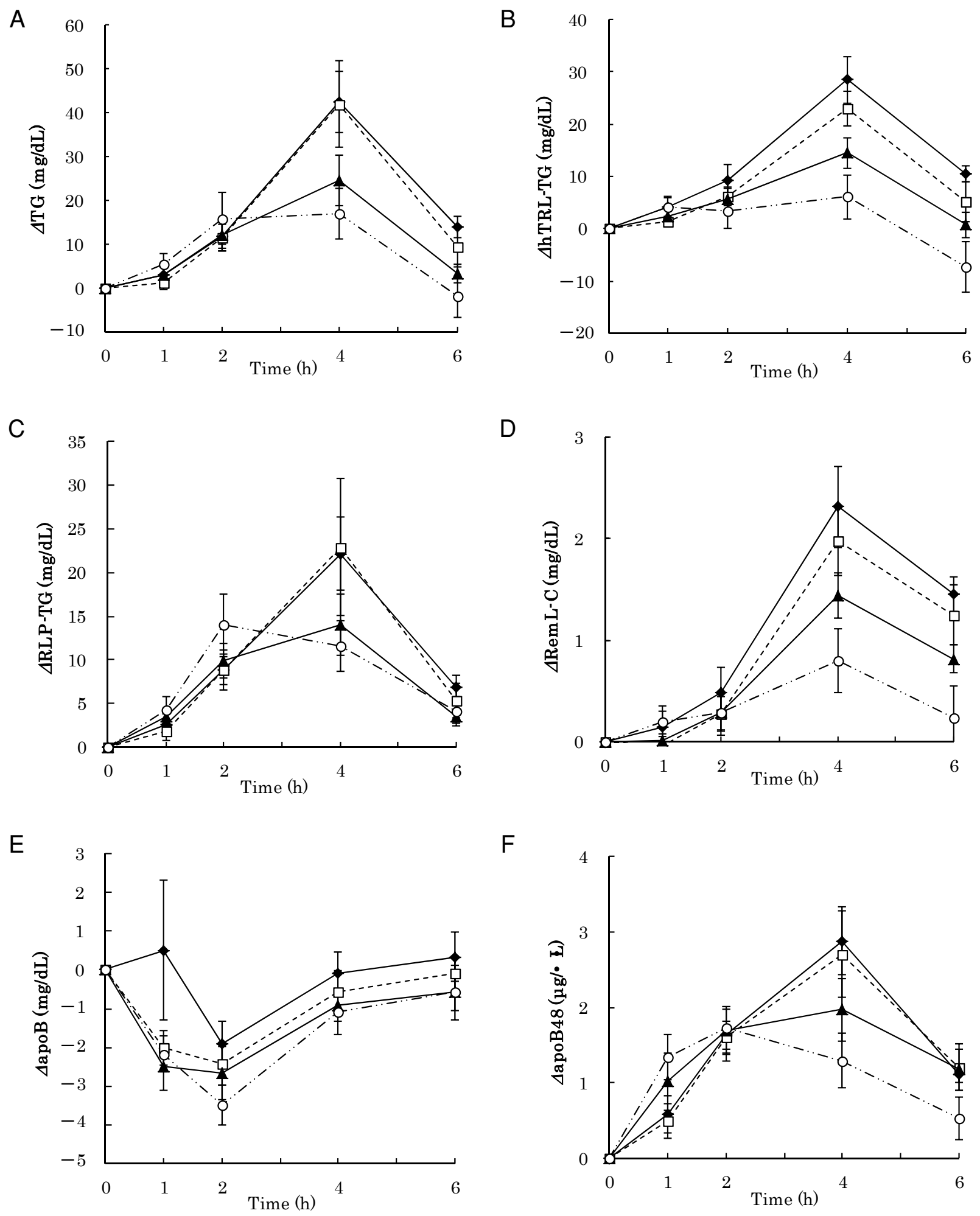

$$
\multimap \text { F100 --ם-- F90G10 —F55G45 - - - G100 }
$$

Fig. 1. Postprandial $\triangle \mathrm{TG}(\mathrm{A}), \triangle \mathrm{hTRL}-\mathrm{TG}(\mathrm{B}), \triangle \mathrm{RLP}-\mathrm{TG}(\mathrm{C}), \triangle \mathrm{RemL}-\mathrm{C}(\mathrm{D}), \Delta \mathrm{apoB}(\mathrm{E})$ and $\Delta$ apoB48 (F). The values are presented as the mean \pm SEM. 
A

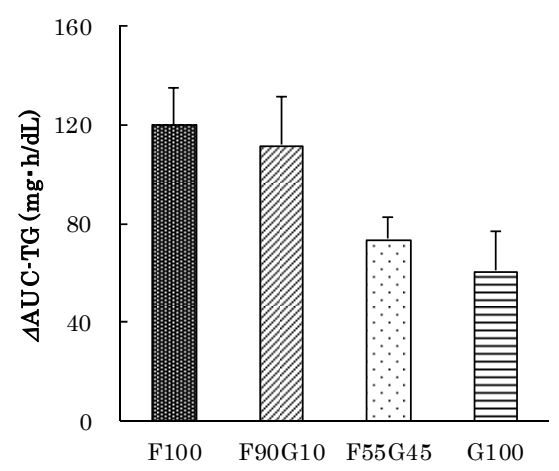

B

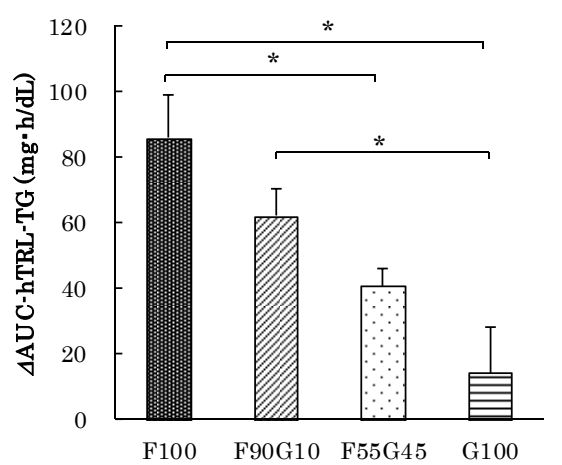

C

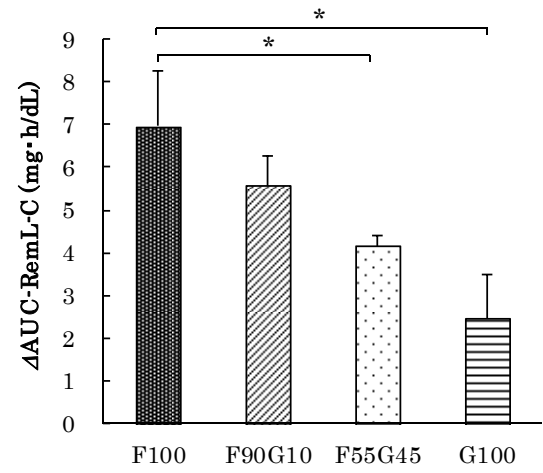

Fig. 2. Incremental area under the curve ( $\triangle \mathrm{AUC})$ for TG (A), hTRL-TG (B) and RemL-C (C). The values are presented as the mean \pm SEM. ${ }^{*} p<0.05$

prandially and that the ratio of fructose to glucose was a key factor in the metabolic disturbance.

Following the ingestion of a high-fructose-containing beverage with fat, the concentration of apoB48 significantly increased after two hours, the peak of which was delayed compared with that observed following the ingestion of glucose with fat. Moreover, in all but the G100 trial, the concentration of apoB48 did not return to baseline at six hours. These results suggest the presence of delayed intestinal absorption of dietary fat and/or the secretion of CM from the intestine in addition to the delayed removal of CM and CM-R, consistent with our previous findings ${ }^{8)}$, although the mechanisms underlying these changes are not clear.

In this study, the serum concentration of apoB (100) was decreased at one to two hours and then returned to baseline at four hours in all trials, suggesting the transient suppression of secretion and/or the stimulation of the removal of very low-density lipoprotein (VLDL). However, in the absence of an increase in the apoB (100) concentration, the concentration of hTRL-TG significantly increased at four hours, except in the G100 trial. Because one apoB100 molecule is present in each VLDL particle, the relationship between hepatic VLDL-TG and VLDLapoB100 secretion allows for estimation of the TG content in the VLDL particles secreted by the liver ${ }^{16)}$. These results suggest the occurrence of an increase in the hepatic TRL particle size without a concurrent increase in number following the ingestion of a fructose- (but not glucose-) containing beverage combined with fat. Although postprandial dyslipidemia has previously been regarded to be a consequence of delayed TRL removal, emerging evidence implicates the intestinal $^{17)}$ and hepatic ${ }^{18)}$ overproduction of TRL as major contributors to postprandial dyslipidemia. The increase in hepatic VLDL secretion may also inhibit the metabolism of CM and CM-R by the liver by competing with exogenous lipoprotein metabolism, as the endogenous and exogenous pathways share the same lipolytic enzyme, lipoprotein lipase (LPL), on the capillary endothelium of extrahepatic tissues, particularly adipose tissue and skeletal muscle ${ }^{19)}$.

Furthermore, it has been reported that a single bolus of fructose rapidly increases hepatic de novo lipogenesis ${ }^{20)}$ and enhances VLDL-TG production ${ }^{21)}$. In the present study, the serum insulin concentrations were lower following the ingestion of fructose with fat versus glucose, and the serum FFA concentrations tended to be suppressed less significantly following the ingestion of fructose rather than glucose. Insulin acutely suppresses FFA release into the circulation from adipose tissue and reduces the flow of substrates for VLDL assembly in the liver ${ }^{22,23}$. Because insulin and glucose also increase the LPL activity in adipose tissue $^{24)}$, the lower insulin and glucose excursion observed following the ingestion of fructose compared with that of glucose may have resulted in the reduced activation of LPL, thus leading to delayed lipolysis of TG in CM and VLDL ${ }^{25)}$ and, eventually, a prolonged increase in the TRL concentration postprandially.

In the present study, the serum lactate concentrations increased at one and two hours in the F100 and F90G10 trials, but not the F55G45 or G100 trials, suggesting that when the ratio of fructose to glucose is significantly high, the serum lactate concentration may increase. Such increased lactate production may occur because the fructokinase activity is increased, the rate-limiting step for glycolysis (phosphofructokinase) is bypassed and the pyruvate kinase activity is stimulated by the accumulation of fructose-1-phos- 
phate ${ }^{26)}$. The increase in the serum concentrations of $\beta$-HB and FFA observed at four and six hours following the ingestion of fructose-containing beverages in this study tended to be smaller than that associated with the glucose-containing beverage. Because $\beta-\mathrm{HB}$ is produced from fatty acids in the liver, the finding of a lower serum FFA concentration is consistent with the smaller increase seen in the serum $\beta$-HB concentration ${ }^{25)}$.

The present results suggest that the ingestion of a high amount of fructose stimulates the synthesis of fatty acids followed by TG-rich, large VLDL in the liver. Our findings also indicate that the higher the ratio of fructose to glucose, the greater the metabolic disturbance, when the same total amount of sugar (fructose and/or glucose) is ingested. Moreover, the lower insulin excursion observed following the ingestion of fructose versus glucose may have also resulted in the reduced activation of LPL, thus leading to delayed TG removal from CM and VLDL. In addition, the lower insulin excursion may have increased the leakage of FFA from the adipose tissue and its supply to the liver. Altogether, we conclude that the ingestion of a high amount of fructose exacerbates the postprandial rise in both exogenous and endogenous lipoproteins.

Nevertheless, there are some limitations associated with this study. Most serious is the specificity of the hTRL-TG (VLDL-TG ${ }^{\mathrm{TM}}$ ) assay, which was developed in the expectation of correctly determining the endogenous TRL-TG concentration ${ }^{15)}$. This assay identifies $100 \%$ of the TG present in VLDL (as expected), $79.0 \%$ (should be $100 \%$ ) in intermediatedensity lipoprotein (IDL), 16.1\% in CM and 29.5\% in LDL (both should be 0\%). In addition, no data were available for CM-R in our study. Therefore, the quantification of endogenous TRL-TG according to this assay may have been inaccurate or not reliable, and the time course of endogenous TRL-TG in the trials should be carefully evaluated. Another weak point is that a relatively small number of subjects was evaluated in this study. These limitations all indicate that the present results should be interpreted with caution.

In summary, we herein demonstrated that the single ingestion of a high-fructose-containing beverage combined with fat cream causes both exogenous and endogenous lipidemia postprandially, even in young healthy women. These foods may thus have even more unfavorable effects on the health of subjects with metabolic syndrome or obesity. Our findings suggest that even the single ingestion of a high-fructose-containing beverage combined with fat induces rapid lipogenesis in the liver, enhances the secretion of TG-rich VLDL and delays the removal of CM and CM-R from the circulation. At present, there are no reliable data regarding the upper limit of the intake of fructose and HFCS for maintaining health in Japanese individu$\mathrm{als}^{27)}$, and the influence of fructose and HFCS ingestion on health remains to be clarified. However, it may be advisable to avoid fructose- and/or HFCScontaining beverages as much as possible in daily life, even among young healthy women.

\section{Conclusion}

The single ingestion of a high-fructose-containing beverage combined with fat cream causes postprandial exacerbation of both exogenous and endogenous lipidemia, even in young healthy women. The ratio of fructose to glucose is a determinant of metabolic disturbance when the sugar load is equicaloric.

\section{Acknowledgements}

This work was supported by Grants-in-Aid for Scientific Research (Basic Research C, 2012) from the Ministry of Education, Culture, Sports, Science and Technology of Japan. We would like to thank Mr. Tatsuhiko Furuhashi for his technical assistance. We also give our thanks to Ms. Kazuno Imura, Ms. Maki Iwatake, Ms. Saki Kanada and Ms. Tomoyo Hukuta for their valuable technical cooperation.

\section{Conflicts of Interest}

None.

\section{References}

1) Ministry of Agriculture, Forestry and Fisheries, Japan, 2012. http://www.maff.go.jp/j/seisan/tokusan/kansho/ pdf/24sy-1.pdf (In Japanese)

2) Ministry of Agriculture, Forestry and Fisheries, Japan, 2008. http://www.maff.go.jp/j/jas/jas_kikaku/kikaku_itiran.html (In Japanese)

3) Ministry of Agricultural, Forestry and Fisheries, Japan, 2012 http://www.famic.go.jp/event/sakuseiiinnkai/kekka/ food/240925/shiryo09.pdf (In Japanese)

4) Bantle JP, Raatz SK, Thomas W, Georgopulos A: Effects of dietary fructose on plasma lipids in healthy subjects. Am J Clin Nutr, 2000; 72: 1128-1134

5) Reiser S, Powell AS, Scholfield DJ, Panda P, Ellwood KC, Canary JJ: Blood lipids, lipoproteins, apolipoproteins, and uric acid in men fed diets containing fructose or high-amylose cornstarch. Am J Clin Nutr, 1989; 49: 832839

6) Zilversmit DB: Atherogenesis: a postprandial phenome- 
non. Circulation, 1979; 60: 473-485

7) Stanhope KL, Griffen SC, Bair BR, Swarbrick MM, Keim NL, Havel PJ: Twenty-four-hour endocrine and metabolic profiles following consumption of high-fructose corn syrup, sucrose, fructose, and glucose sweetened beverages with meals. Am J Clin Nutr, 2008; 87: 1194-1203

8) Saito H, Kagaya M, Suzuki M, Yoshida A, Naito M: Simultaneous ingestion of fructose with fat exacerbates postprandial exogenous lipidemia in young healthy Japanese women. J Atheroscler Thromb, 2013; 20: 591-600

9) Latulippe ME, Skoog SM: Fructose malabsorption and intolerance: effects of fructose with and without simultaneous glucose ingestion. Crit Rev Food Sci Nutr, 2011; 51: 583-592

10) Nabeno Y, Fukuchi Y, Matsutani Y, Naito M: Influence of aging and menopause on postprandial lipoprotein responses in healthy adult women. J Atheroscler Thromb, 2007; 14: 142-150

11) Nabeno-Kaeriyama Y, Fukuchi $Y$, Hayashi S, Kimura T, Tanaka A, Naito M: Delayed postprandial metabolism of triglyceride-rich lipoproteins in obese young men compared to lean young men. Clin Chim Acta, 2010; 411: 1694-1699

12) Hashimoto S, Ootani K, Hayashi S, Naito M: Acute effects of shortly pre-versus postprandial aerobic exercise on postprandial lipoprotein metabolism in healthy but sedentary young women. J Atheroscler Thromb, 2011; 18: 891-900

13) Hashimoto S, Hayashi S, Yoshida A, Naito M: Acute effects of postprandial aerobic exercise on glucose and lipoprotein metabolism. J Atheroscler Thromb, 2013; 20: 204-213

14) Matthews DR, Hosker JP, Rudenski AS, Naylor BA, Treacher DF, Turner RC: Homeostasis model assessment: insulin resistance and beta-cell function from fasting plasma glucose and insulin concentrations in man. Diabetologia, 1985; 28: 412-419

15) Okada M, Saito T, Yoshimura H, Noguchi Y, Ito T, Sasaki H, Hama H: Surfactant-based homogeneous assay for the measurement of triglyceride concentrations in VLDL and intermediate-density lipoprotein. Clin Chem, 2005; 51: $1804-1810$
16) Magkos F, Patterson BW, Mohammed BS, Klein S, Mittendorfer B: Women produce fewer but triglyceride-richer very low-density lipoproteins than men. J Clin Endocrinol Metab, 2007; 92: 1311-1318

17) Hsieh J, Hayashi AA, Webb J, Adeli K: Postprandial dyslipidemia in insulin resistance: mechanisms and role of intestinal insulin sensitivity. Atheroscler Suppl, 2008; 9: 7-13

18) Sparks JD, Sparks CE, Adeli: Selective hepatic insulin resistance, VLDL overproduction, and hypertriglyceridemia. Arterioscler Thromb Vasc Biol, 2012; 32: 2104-2112

19) Brunzell JD, Hazzard WR, Porte D Jr, Bierman EL: Evidence for a common, saturable, triglyceride removal mechanism for chylomicrons and very low density lipoproteins in man. J Clin Invest, 1973; 52: 1578-1585

20) Parks EJ, Skokan LE, Timlin MT, Dingfelder CS: Dietary sugars stimulate fatty acid synthesis in adults. J Nutr, 2008; 138: 1039-1046

21) Hudgins LC, Parker TS, Levine DM, Hellerstein MK: A dual sugar challenge test for lipogenic sensitivity to dietary fructose. J Clin Endocrinol Metab, 2011; 96: 861-868

22) Malmström R, Packard CJ, Caslake M, Bedford D, Stewart P, Yki-Järvinen $\mathrm{H}$, Shepherd J, Taskinen MR: Effects of insulin and acipimox on VLDL1 and VLDL2 apolipoprotein B production in normal subjects. Diabetes, 1998; 47: 779-787

23) Lewis GF, Uffelman KD, Szeto LW, Steiner G: Effects of acute hyperinsulinemia on VLDL triglyceride and VLDL apoB production in normal weight and obese individuals. Diabetes, 1993; 42: 833-842

24) Wang H, Eckel RH: Lipoprotein lipase: from gene to obesity. Am J Physiol Endocrinol Metab, 2009; 297: E271-E288

25) Chong MF, Fielding BA, Frayn KN: Mechanisms for the acute effect of fructose on postprandial lipemia. Am J Clin Nutr, 2007; 85: 1511-1520

26) Hallfrisch J: Metabolic effects of dietary fructose. FASEB J, 1990; 4: 2652-2660

27) National Health and Nutrition Survey, Ministry of Health, Labour and Welfare, Japan, 2010. http://www.mhlw. go.jp/bunya/kenkou/sessyu-kijun.html (In Japanese) 